\title{
Planificación de escenarios: una herramienta estratégica para el análisis del entorno
}

\author{
Yori Conill, Ligia * \\ Hernández de Velazco, Judith ** \\ Chumaceiro Hernández, Ana ***
}

\begin{abstract}
Resumen
El presente artículo tiene como objetivo establecer la utilidad de la planificación de escenarios dentro del contexto estratégico de las organizaciones. Con esta finalidad se sustenta en las teorías propuestas por Bas (2002); Frances (2001); Saloner et al. (2005); Dess y Lumpkin (2003); Garrido (2003), entre otros. La metodología utilizada es documental o bibliográfica. Esta técnica de dirección permite redefinir y actualizar estrategias opcionales, por medio de la evaluación de los riesgos y oportunidades provenientes del entorno, como también plantear planes contingentes ajustados a una realidad determinada. Se concluye que la planificación de escenarios posee una importancia relevante dentro del contexto estratégico (internacional y de Venezuela), porque el mismo se nutre de un estudio sistemático del entorno y constituye una herramienta de la planificación estratégica orientada a enfrentar la incertidumbre del futuro y a optimizar la toma de decisiones a través del desarrollo de modelos de simulación.
\end{abstract}

Palabras clave: Planificación de escenarios, herramienta estratégica, entorno, incertidumbre.

Recibido: 06-12-10. Aceptado: 20-05-11

* Doctora en Ciencias Sociales - Mención Gerencia (URBE). Magíster Scientarium en Gerencia Financiera (UNERMB). Economista (LUZ). Profesora Titular UNERMB. Investigadora activa del Centro de Estudios e Investigaciones Socioeconómicas y Políticas (CEISEP). E-mail: ligiayori@hotmail.com

** Doctora en Ciencias Sociales - Mención Gerencia (URBE). MSC Administración de Empresas. Licenciada en Ciencias Políticas y Administrativas. Profesora Titular UNERMB. Coordinadora General del Centro de Estudios e Investigaciones Socioeconómicas y Políticas (CEISEP).

E-mail: lasanas23@cantv.net

*** Especialista en Gerencia Tributaria. Licenciada en Administración - Mención Gerencia Industrial. Docente. Investigadora Novel del Centro de Estudios e Investigaciones Socioeconómicas y Políticas (CEISEP). E-mail: ceciliahernanz@hotmail.com 


\title{
Planning Scenarios: A Strategic Tool for Analyzing the Surroundings
}

\begin{abstract}
The objective of this article is to establish the usefulness of planning scenarios within the strategic context of organizations. The study is supported on theories proposed by Bas (2002); Frances (2001); Saloner et al (2005); Dess and Lumpkin (2003); and Garrido (2003), among others. Methodology is documentary or bibliographic. This management technique makes it possible to redefine and update optional strategies by evaluating risks and opportunities coming from the surroundings, as well as proposing contingent plans adjusted to a certain reality. Conclusions are that planning scenarios has a relevant importance within the international and Venezuelan strategic context, because this strategy is nurtured by a systematic study of the surroundings and constitutes a strategic planning tool oriented toward facing the uncertainty of the future and optimizing decision making through the development of simulation models.
\end{abstract}

Key words: Planning scenarios, strategic tool, surroundings, uncertainty.

\section{Introducción}

La actual concepción de la dirección estratégica considera el estudio del entorno o medio externo como elemento determinante para la formulación de estrategias a seguir. En efecto, en los tiempos de cambio, la pertinencia y calidad de la información ambiental se torna una necesidad apremiante para que las organizaciones sean más competitivas en el mercado y logren alcanzar sus objetivos organizacionales.

El desarrollo de esta técnica supone pronosticar el entorno y desarrollar proyecciones posibles sobre el alcance y velocidad de los cambios del medio. Así mismo, la planificación de escenarios proporciona ideas sobre como pueden los directivos ser mas creativos a la hora de anticipar el futuro a través de la imaginación de amenazas y oportunidades futuras.

Bajo estas consideraciones, los escenarios deberán ser utilizados en los negocios cuyo entorno es proclive a cam- bios fundamentales o repentinos, o en los que la anticipación de tales cambios resulta de importancia estratégica. De allí deriva su importancia como técnica enmarcada dentro de la predicción social que es aplicada cada vez por un mayor contingente de negocios o sectores del mundo.

Atendiendo a estas reflexiones surge la relevancia de este artículo, la cual coloca especial énfasis en la necesidad que posee toda organización de estudiar el entorno y así optimizar las posibilidades de supervivencia de las empresas a través de la aplicación de herramientas como la planificación de escenarios, cuya utilización se ha extendido exitosamente en los últimos años.

\section{La planificación de escenarios en el ámbito venezolano}

En primera instancia, resulta importante resaltar que el desarrollo de esta 
Planificación de escenarios: una herramienta estratégica para el análisis del entorno Yori Conill, Ligia; Hernández de Velazco, Judith y Chumaceiro Hernández, Ana

técnica cualitativa generalmente se recomienda para países como Venezuela, que no cuentan con una buena base de datos y donde las variables macro presentes en el escenario económico político y social resultan relativamente inestables. En torno a estas consideraciones el Centro de Estudios del Desarrollo de la Universidad Central de Venezuela (CENDES - UCV) (2009) plantea que las dificultades creadas por la carencia de datos básicos sistemáticos y actualizados en nuestro país y en el ámbito latinoamericano, apuntan hacia la necesidad del uso de metodologías prospectivas con métodos y técnicas adecuadas a nuestras realidades.

Al respecto Francés (2001) expresa que en el ámbito venezolano los escenarios macroeconómicos nacionales se desarrollan tomando en consideración lo que acontece en el mercado petrolero mundial y en materia de política económica del gobierno nacional. Es así como Petróleos de Venezuela, la Banca Nacional y otros entes corporativos, disponen de una unidad de estudios económicos y elaboran sus predicciones en torno a estas variables.

Sin embargo, en la mayoría de los casos la gerencia utiliza escenarios económicos elaborados por empresas e instituciones especializadas, debido a la escasa tradición en materia de planificación que posee el gerente venezolano, el cual desarrolla los escenarios generalmente en un ambiente colmado de incertidumbre y de marcada inestabilidad, lo cual constituye una limitante para determinar oportunidades y amenazas en el entorno $y$, por ende, desarrollar escenarios realistas y factibles de alcanzar.
También plantea Francés (2001) que en la construcción de escenarios el gerente no es capaz de prever los acontecimientos inevitables en el contexto del panorama económico, político, social lo cual constituye una condición necesaria para el desarrollo de esta técnica de predicción social y, por consiguiente para la elaboración de escenarios situacionales.

A los argumentos anteriormente expuestos, se agrega el hecho de la escasa predicción por parte de la gerencia venezolana para proyectar variables poco cuantificables necesarias para el desarrollo de escenarios. En forma análoga, la gerencia venezolana se encuentra desprovista de un carácter anticipatorio, proactivo y con amplia visión de futuro que permita prever contingencias en caso de que falle el escenario elegido.

En efecto, las organizaciones con una mentalidad anticipatoria que adoptan una actitud proactiva, son las que dispondrán de un horizonte mayor proporcionado por una mayor cantidad de información, que les permitirá tener mayores posibilidades de sobrevivir al correr de los tiempos y superar a sus competidores.

En este orden de ideas Bas (2002) expresa que no resulta descabellado pensar que tan solo las organizaciones flexibles y que adopten una actitud abierta en la gestión, orientada hacia el futuro, tendrán mas posibilidades de no quedar obsoletas por ineficaces, ni ser anuladas por la competencia en un entorno tan cambiante y competitivo como el presente y que, definitivamente, permite acercarse con cierta precisión a un futuro cargado de incertidumbre.

Es así como la aplicación del método de los escenarios para la construcción 
prospectiva de la planificación en Venezuela según la Fundación para el Desarrollo de la Región Centro Occidental de Venezuela (FUDECO) (2009) se sustenta en tres (3) elementos expresados en la Ley Orgánica de Planificación:

1. Las condiciones iniciales: información suministrada por el diagnóstico, base para alcanzar la imagen deseada y que caracteriza la realidad del país al momento de efectuar la planificación.

2. Las imágenes objetivo: Imágenes de los futuros imaginados o proposiciones deseables a futuro propuestos para un periodo determinado.

3. Trayectoria estratégica: dirección de las acciones y medidas para el traslado de la base inicial (presente o condiciones iniciales) hasta la base final (al futuro o imágenes objetivo). Estas se componen de acciones estructurantes o prioridades, acciones de acompañamiento (inducidas) y las acciones no compatibles (acciones a evitar si se desea alcanzar el futuro).

No obstante, esta técnica prospectiva debe ser utilizada con cautela para alcanzar las aspiraciones anteriormente expuestas, tomando en consideración las necesidades reales presentes en nuestro medio. Al respecto, como una contribución para disminuir las dificultades relativas a la efectividad metodológica en el uso de la prospectiva, el CENDES - UCV ha incluido en sus programas el curso "Prospectiva: Construcción de escenarios para enfrentar la incertidumbre", con el apoyo de las nuevas tecnologías de información y comunicación, entre otras, expresiones de interés que se han producido para facilitar la aplicación de este método gerencial.
En torno a estos señalamientos, cabe resaltar que cuanto mayor es el grado de información que se posea y mayor la potencia de las herramientas de análisis y predicción, mayor es también la dificultad para hacer predicciones del futuro de manera acertadas debido a la magnitud y las dimensiones de los cambios del entorno, aunado a la incertidumbre presente en el medio que en algunas oportunidades tornan poco efectivas la utilización de las técnicas de predicción mas avanzadas. En efecto, la velocidad de los cambios y el nivel de imprecisión presentes en el entorno, es en la actualidad de tal magnitud que difícilmente se acierta en predicciones a largo e incluso a mediano plazo.

\section{Importancia del análisis del entorno}

Las dimensiones de los cambios económicos, sociales, culturales, políticos, gubernamentales, tecnológicos y competitivos, justifican la necesidad de un análisis del entorno efectivo, permitiendo a las organizaciones formular $y$ ejecutar estrategias en forma idónea, mediante la evaluación de las circunstancias que rodean las tendencias, hechos, así como la forma en que ellas producirán un impacto significativo sobre la empresa.

Los argumentos expuestos permiten inferir que el análisis del entorno constituye un requisito indispensable para realizar efectivamente la planeación y gestión estratégica debido a la gran cantidad de factores involucrados. Al respecto Serna (2001) plantea que tales factores incluyen tanto oportunidades como amenazas y tienen que ser considerados en el proceso de planeación y gestión 
Planificación de escenarios: una herramienta estratégica para el análisis del entorno Yori Conill, Ligia; Hernández de Velazco, Judith y Chumaceiro Hernández, Ana

empresarial, habilitando así a la empresa a reaccionar oportunamente ante los factores externos.

Estas consideraciones permiten inferir que resulta imprescindible la urgencia de mantener un permanente examen sobre los factores externos que afectan o condicionan el éxito de una empresa, los cuales introducen una marcada incertidumbre a la hora de tomar decisiones estratégicas.

En forma análoga, Dess y Lumpkin (2003) expresan que una buena exploración del entorno alerta a las organizaciones sobre tendencias y acontecimientos críticos antes que los competidores lo reconozcan. De lo contrario, la empresa puede verse forzada a adoptar una postura reactiva en lugar de proactiva, esta última posición deseable para alcanzar niveles eficientes de desempeño.

En correspondencia con lo anteriormente expresado, Certo y Peter (1996) señalan que el éxito o fracaso de una organización dependerá del grado de exactitud con que la alta dirección lea o interprete el ambiente y de la eficacia con que responda a esa lectura. Por consiguiente, la alta dirección debe dedicar gran parte de su tiempo y de sus esfuerzos a reunir y analizar datos relacionados con los factores ambientales que consideren importantes.

Bajo esta perspectiva, la efectividad del análisis ambiental va a depender de la habilidad para enfrentar oportunamente en forma dinámica y acelerada los desafíos del cambio, tales como los cambios del gusto del consumidor, de las condiciones políticas, de la estructura del mercado, así como los cambios tecnológicos, que no sólo pueden tener efectos en una u otra compañía individualmente, sino que pueden generar la crisis o el repunte de toda una industria.

En efecto, la complejidad de los factores anteriormente citados aunado al aumento de la competencia a partir de la globalización han provocado un aumento de la incertidumbre en el mercado, lo cual ha conllevado a la alta gerencia a ampliar sus horizontes de planificación y a tomar decisiones bajo grado de incertidumbre cada vez mayores. En este sentido, la acción gerencial debe apoyarse sobre un estudio sistemático y minucioso del medio ambiente, como elemento vital para la formulación de estrategias y por ende optimizar el proceso de toma de decisiones.

En correspondencia con lo expresado anteriormente, Duane (2001) señala que la calidad de las decisiones se encuentra íntimamente ligada a la disponibilidad y veracidad de información útil en el momento en que se tome la decisión. Esta definición destaca la relevancia que tiene la información para cualquier tipo de negocio; por ello se presume que las decisiones en las organizaciones se toman basadas en la información proveniente del medio ambiente que debe ser precisa, pertinente y efectiva, por cuanto las mismas tienen un impacto directo en relación a costos, prestigio, imagen, satisfacción de necesidades a sus grupos de interés y, específicamente, en la definición de estrategias.

Al respecto, Hitt et al. (2004) expresan que definitivamente el entorno influye en las opciones estratégicas que la empresa tiene a su alcance, así como en las decisiones que pueda tomar a la luz de ellas. En este sentido, las empresas que deseen alcanzar la competitividad estratégica deben 
tener conciencia de las distintas dimensiones del entorno y también entenderlas e interpretarlas oportunamente.

Por otro lado, Garrido (2006) plantea que la actual concepción de la dirección estratégica de las organizaciones considera el estudio del entorno o medio externo como algo indisolublemente ligado al diseño y formulación de la estrategia a seguir, así como la necesaria observación para el desarrollo y seguimiento de la línea de actuación elegida.

Es esta progresiva importancia de la información y la importancia de analizar las variables ambientales claves, lo que justifica que muchos directivos dediquen una elevada parte de su tiempo a buscar herramientas que le permitan obtener información, sobre todo aquella que resulta difícil de obtener con el propósito de optimizar la consecución de los objetivos organizacionales.

Reconocida la necesidad de tomar en consideración el entorno como factor explicativo del acontecer en las organizaciones, no cabe otra alternativa para una nueva gestión que incorporar la observación y el análisis del entorno como elemento necesario para que las organizaciones sobrevivan en el mercado.

\section{Planificación de escenarios}

Debido a la relevancia que reviste la planificación de escenarios y la marcada difusión de los mismos en los últimos años como herramienta estratégica para analizar el entorno y, por ende, reducir la incertidumbre, resulta imprescindible realizar algunas definiciones al respecto.

En este sentido, Bas (2002) plantea que un escenario es un futurible, un futuro posible de entre varias alternativas, que describe una situación hipotética futura. En forma análoga, Francés (2001) expresa que los escenarios son descripciones de los posibles entornos en los cuales podría ubicarse la empresa en el futuro, ya que la función principal de los escenarios es alertar acerca de posibles eventos que puedan afectar la empresa.

Por otro lado Garrido (2003) plantea que dicho método no es otra cosa que la consideración de varias hipótesis de futuros diferentes y alternativas a la hora de valorar el mismo, permitiendo contemplar mas posibilidades de futuro que la descrita por una determinada técnica de predicción, al observar bajo distintos entornos, cuales serán los puntos débiles o fuertes, las amenazas y las oportunidades con el fin de prepararse al respecto.

Por su parte David (2003) señala que los escenarios se pueden describir como conjuntos alternativos de posibles ocurrencias futuras donde se anticipa el impacto de diversos argumentos en una organización, erigiéndose esta probablemente como la técnica más popular de la predicción social.

Dentro de este orden de ideas, Saloner et al. (2005) plantean que un escenario es un futuro posible para la industria que posee coherencia interna, es decir, es un panorama plausible de cómo podría ser la industria en el futuro.

En virtud de los argumentos expuestos se puede inferir que los escenarios constituyen un instrumento que ayuda a los responsables de la decisión a crear un contexto para la planificación y programación, permitiendo elegir entre opciones de desarrollo. Así mismo, construir repre- 
Planificación de escenarios: una herramienta estratégica para el análisis del entorno Yori Conill, Ligia; Hernández de Velazco, Judith y Chumaceiro Hernández, Ana

sentaciones de los fututos posibles, a través de la combinación de datos cuantitativos y cualitativos, así como el camino que conduce a su consecución permitiendo establecer estrategias futuras.

Los conceptos anteriormente desarrollados comprueban que la planificación de escenarios constituye un instrumento para la toma de decisiones que permite manejar y predecir ambientes de rápido cambio social y de interacciones complejas, disminuyendo el nivel de incertidumbre a través de la descripción de futuros alternativos posibles. En efecto, el estudio del futuro es consecuencia directa de la incertidumbre, ya que cuanto mayor sea el nivel de ésta, mayor será el interés por conocer el futuro.

Cabe señalar que mientras el entorno era relativamente estable, las técnicas de previsión como la extrapolación de tendencias eran suficientemente buenas y validas. En este sentido, bastaba con proyectar las tendencias a años sucesivos para tener una idea aproximada de hacia donde se dirigían las cosas.

En la actualidad, las técnicas clásicas se han revelado como insuficientes para predecir el futuro, sobre todo en algunos sectores y sociedades. Así mismo, la velocidad e importancia de las variaciones hace necesaria una constante observación; de ahí la proliferación de institutos dedicados a la predicción y a la aparición de nuevas técnicas, destacándose entre ellas, el método de los escenarios.

La aplicación sistemática de este método como herramienta de previsión del futuro no tiene lugar hasta mediados del siglo XX. Al respecto, Bas (2002) expresa que fue precisamente durante este siglo cuando se han desarrollado y es- tructurado lo que se ha dado en llamar estudios del futuro.

El autor también señala que ha sido el industrialismo y el desarrollo del sistema capitalista, y sus consecuencias, los factores que han propiciado en última instancia la aparición de los estudios del futuro. Definitivamente, la depresión de los años veinte constituyó el eslabón que orientó a los analistas económicos en la búsqueda de modelos econométricos que previeran catástrofes de este tipo.

Pero es a final de la segunda guerra mundial, el hito histórico que marca el origen del desarrollo y consolidación de los estudios del futuro donde las economías devastadas por la guerra requerían una planificación que exigía a su vez y previamente un ejercicio prospectivo considerable. A esto se agrega la tensión que origina la guerra fría, que conlleva a que los analistas de política exterior estadounidenses intentaran prever cuales podrían ser los movimientos del enemigo. Esto definitivamente conllevó al planteamiento de futuros hipotéticos o posibles escenarios que facilitarán la previsión.

En este sentido, Bas (2002) señala que es Herman Kahn, gurú de la planificación estratégica durante los años cincuenta y sesenta, el primero en utilizar este método de forma continuada, por tanto, también fue el responsable de la introducción de este término dentro del contexto de los estudios del futuro.

En efecto, el método de escenarios comenzó a ser utilizado a mediados de los años cincuenta, dentro de los estudios estratégicos y militares desarrollados por la Rand Corporación, en la que trabajaba Kahn para el gobierno de Estados Unidos $\mathrm{y}$, mas concretamente, para la planifica- 
ción estratégicas de la Fuerza Aérea de los Estados Unidos de Norteamérica.

En los estudios señalados se trataba de identificar senderos de actuación alternativos y sus hipotéticos resultados en contextos diferentes, con el fin de orientar la toma de decisiones, previniendo las posibles consecuencias de éstas para el orden mundial. Cabe resaltar que Kahn no asignaba probabilidad de ocurrencia a los escenarios considerados, sino que simplemente trataba de anticipar escenarios futuros dejando de lado el pronóstico.

Bas (2002) también señala que la primera aplicación del método de escenarios en el ámbito de la empresa privada fue desarrollada para General Electric, entre finales de los años sesenta y principios de los setenta, con el fin de caracterizar a la sociedad y la economía norteamericana de los años ochenta.

Sin embargo, la consolidación del método de escenarios como herramienta prospectiva y estratégica aplicada a la toma de decisiones empresarial, viene con la adopción de la multinacional Shell Internacional a finales de los años setenta, a través de la adopción de este método en las rutinas de trabajo de gestión, en lugar de la predicción tradicional para diseñar la estrategia corporativa.

Bajo esta perspectiva, el método de escenarios se ha revelado sumamente productivo, al permitir a la empresa adaptarse a los cambios y situaciones contingentes al aprovechar las posibilidades latentes en éstos, confiriéndole en muchos casos una ventaja competitiva, erigiéndose en una técnica que se ha comprobado consistentemente efectiva para enfrentar la incertidumbre estratégi- ca en numerosas compañías, en diversas industrias.

Al respecto, Dess y Lumpkin (2003) plantean que la planificación de escenarios se mueve entre un abanico de disciplinas y temas, entre ellos la economía, la psicología, la sociología y la demografía. Refiere el autor que esta técnica normalmente comienza con un debate sobre lo que los participantes piensan respecto a las tendencias sociales, económicas, políticas y tecnológicas que puedan afectar a la materia en estudio.

En torno a estas consideraciones, los escenarios deberían ser utilizados en los negocios cuyo entorno es proclive a cambios fundamentales o repentinos, o en los que la anticipación a tales cambios es de vital importancia estratégica. Tales condiciones se aplican cada vez a un mayor número de negocios o sectores del mundo.

Partiendo de las evidencias anteriores, se puede inferir que un escenario ha de cumplir simultáneamente tres condiciones: pertinencia, coherencia y verosimilitud, y puede ser descrito como un instrumento que ayuda a la toma de decisiones, al proveer un contexto para la planificación y la programación, bajando el nivel de incertidumbre y aumentando el nivel de conocimiento, en relación a las consecuencias de las acciones, que han sido o van a ser emprendidas en el presente.

También esta técnica constituye una vía alternativa idónea y completa de reinterpretar y reorganizar la información recabada a través de otras técnicas, ya sean anticipatorias (Delphi), matriz de impacto cruzado, análisis de series temporales, entre otras. 
Planificación de escenarios: una herramienta estratégica para el análisis del entorno Yori Conill, Ligia; Hernández de Velazco, Judith y Chumaceiro Hernández, Ana

\section{Enfoques para construir escenarios}

Existen diversos enfoques acerca de la construcción de escenarios que, aunque coinciden en lo básico, divergen en algunos puntos. En tal sentido, existen diversas propuestas elaboradas por futurólogos del mundo que han trabajado con diferentes enfoques y en diversos ámbitos. A continuación se tomará como referencia el enfoque planteado por Bas (2002), el cual considera los siguientes pasos en la elaboración de un escenario:

- Decidir el sistema sobre el cual se va a trabajar (variables y relaciones)

- Decidir el espacio temporal para los escenarios (plazo de ocurrencia)

- Decidir cuantos escenarios se quieren construir (tres o cuatro es lo idóneo).

- Determinar las variables principales que van a estructurar los escenarios y discutir las asunciones sobre el futuro.

- Definir el valor que van a tomar las variables en cada escenario considerado.

- Decidir eventos específicos que pueden ocurrir durante el espacio temporal de los escenarios.

- Crear un título de trabajo para cada escenario.

- Esbozar los escenarios.

- Revisar / evaluar el borrador en base a los siguientes seis criterios: credibilidad, comprensibilidad, consistencia interna, interés, ajuste a los objetivos prefijados y exhaustividad.

No obstante estos pasos pueden variar y siguiendo el criterio desarrollado por el autor anteriormente citado lo concibe de la siguiente manera identificación del tema focal, entre ellos los objetivos del plan, el horizonte temporal así como también los participantes adecuados.

- Analizar los efectos del entorno.

- Identificar y caracterizar los factores de cambio.

- Validar los factores de cambio.

- Clasificar las variables para establecer los factores claves de cambio más importantes.

- Identificar la incertidumbre de las variables críticas.

- Selección de escenarios lógicos.

- Desarrollo de escenarios.

- Evaluación de la coherencia de escenarios.

- Evaluación de las implicaciones de los escenarios desarrollados con respecto a los objetivos estratégicos.

En este orden de ideas, Garrido (2003) expresa que el método de escenarios supone:

- Definir las variables o factores a tener en cuenta (los factores del medio externo, cuya evolución se considera relevante para planificar).

- Establecer hipótesis de comportamiento para cada una de esas variables.

- Fijar tendencias y acontecimientos futuros en términos de probabilidades.

- Construir los posibles escenarios factibles y realistas.

- Diseñar el perfil del entorno para cada escenario posible y evaluar su impacto sobre la organización para la cual se realiza la observación. 


\subsection{Tipos de escenarios}

Sobre la base de las ideas expuestas, los escenarios pueden ser formulados para diferentes ámbitos geográficos, entre ellos, global, regional, continental, nacional, provincial, local. En cuanto a su horizonte temporal, existe una amplia gama de posibilidades, ya sea uno, tres, cinco, diez y veinte años. Al respecto, Francés (2001) afirma que el horizonte a adoptar, debe coincidir con el análisis de la estrategia a realizar, clasificándolo de la siguiente manera:

\section{a) Escenarios de largo plazo}

En este escenario se identifican una serie de variables relevantes, cuyo comportamiento proporciona un perfil del entorno nacional. Estas variables se pueden clasificar en físico-ambientales, técnico-económico, sociales y político- institucionales.

Siguiendo esta metodología para los tres tipos de variables descritas, se pueden definir tres estados y calificarlos en general como: optimista, pesimista y probable. Al respecto Francés (2001) plantea que es posible utilizar el panel de expertos para asignar probabilidades de ocurrencia a los tres estados de cada variable, refiriendo también que el escenario más probable quedaría conformado por el conjunto de opciones consideradas más probables en cada variable.

El autor citado también refiere que la escogencia de un escenario mas probable implica determinados supuestos acerca de las fuerzas que operan dentro de un país analizado, como en su marco institucional, así también se pueden generar escenarios alternos combinando aquellas opciones que resulten coherentes.

\section{b) Escenarios situacionales}

La construcción de estos escenarios se efectúa a partir de las fuerzas motoras que pueden ser identificadas en el entorno. Bajo este contexto, Francés (2001) señala que las fuerzas motoras son los elementos que mueven la trama y que determinan el resultado.

Este tipo de análisis de escenarios fue desarrollado por el grupo de planificación de la empresa petrolera Anglo Holandesa Shell en la década de 1970. En este sentido, el equipo de Shell logró anticipar la crisis petrolera de comienzos de la década, examinando fuerzas motoras como el enfrentamiento Árabe-Israelí en el medio oriente y el incremento de la demanda mundial de gasolina. Por otro lado, también anticiparon la caída de los precios del petróleo a comienzos de la década de 1980, lo cual permitió mejorar su posicionamiento dentro de la industria petrolera global.

Entre las fuerzas motoras necesarias para el desarrollo de este tipo de escenarios se encuentra: el comportamiento de los mercados, de productos, de insumos, financieros, nacionales e internacionales, las tendencias demográficas, los cambios tecnológicos, las políticas gubernamentales, las actitudes sociales y las relaciones internacionales.

Francés (2001) plantea que al construir escenarios resulta importante identificar los acontecimientos inevitables, como el envejecimiento de la población o el agotamiento de los yacimientos minerales sin explotación, allanando así, el camino para establecer las áreas esenciales de incertidumbre. Así también estos escenarios aportan una 
Planificación de escenarios: una herramienta estratégica para el análisis del entorno Yori Conill, Ligia; Hernández de Velazco, Judith y Chumaceiro Hernández, Ana

forma refinada de plantear opciones explorando los diferentes resultados posibles, si los acontecimientos toman diferentes rumbos.

Con referencia a lo planteado, la formulación y análisis de escenarios situacionales se pueden realizar en los ámbitos corporativos y de negocios, y sus resultados permiten identificar oportunidades y amenazas en el entorno; son útiles para generar estrategias.

c) Escenarios macroeconómicos de mediano plazo.

Para el horizonte de corto a mediano plazo se trabaja generalmente con escenarios macroeconómicos, en los cuales las variables de desempeño económico describen el escenario. Este tipo de análisis, resalta Francés (2001), es válido en general para los países desarrollados y aquellos en desarrollo, donde la política económica es relativamente estable; y se pueden formular tres escenarios: positivos, negativos y probable.

No obstante, cabe resaltar que en los países donde la política económica esta sujeta a marcadas variaciones, como ha sido el caso de Venezuela y de algunos países latinoamericanos en los últimos años, los distintos escenarios responden a diferentes orientaciones de política económica pero los mismos siguen siendo más o menos probables.

Entre las variables de comportamiento que describen los escenarios macroeconómicos destacan: tasa de cambio, tasa de inflación, tasa de interés activa y pasiva, crecimiento del PIB, balanza de pagos, tasa de desempleo, nivel de reserva internacional.

\section{La planificación de escenarios una herramienta estratégica}

A lo largo del siglo $X X$ y comienzos del nuevo siglo, el ritmo de cambios acelerados que ha confrontado la sociedad en materia tecnológica y económica esta obligando a las organizaciones a asumir un proceso de cambio continuo, en una permanente búsqueda de mejorar la competitividad. De hecho, actualmente nadie coloca en duda la validez de que la información es poder, por lo cual no es extraño observar como cada vez mayor número de organizaciones disponen de todos los medios necesarios para su obtención y control.

Una de estas técnicas utilizadas para predecir el entorno lo constituye la planificación de escenarios, la cual se encuentra diseñada para ayudar al director o gerente estratégico a identificar en el medio estos factores que contribuyen a incrementar la incertidumbre que circunda y afecta de manera directa a la organización.

En este sentido, la planificación de escenarios se erige como una herramienta estratégica ya que le permite a la organización mantener informado al gerente de todos los cambios que se susciten en materia económica, política, social, competitiva y tecnológica que acontecen en el entorno.

Bajo esta perspectiva, esta técnica se convierte en una necesidad vital ante los cambios veloces registrados en el medio y en una herramienta estratégica donde las organizaciones asumen una actitud netamente proactiva con el propósito de obtener información oportuna y de calidad a través de la predicción de variables que 
le permita a las organizaciones ser mas competitivas dentro de un contexto cada vez mas cambiante y turbulento.

En torno a estos señalamientos David (2003), señala que una vez seleccionada las variables ambientales claves y ubicadas las fuentes específicas de información, se procede a predecir las variables ambientales las cuales constituyen presunciones cultas sobre tendencias y eventos futuros.

Al respecto, Thompson y Strickland (2004) plantean que cuando los analistas pronostican, hacen proyecciones viables de lo que podría ocurrir como consecuencia de los cambios y las tendencias detectadas mediante la búsqueda y el monitoreo, pero también cuando ocurrirán.

Estas consideraciones revelan que la predicción es una actividad muy compleja debido a la variedad de factores interrelacionados que influyen en ella, como son: los cambios de la situación política, las innovaciones tecnológicas, los cambios culturales, los nuevos productos, la mejora de los servicios, los competidores, los cambiantes valores sociales, la variable situación económica y los eventos no predecibles. Se trata de una actividad tan compleja que muchos estrategas se basan en predicciones ambientales publicadas para identificar de manera efectiva las oportunidades y amenazas claves y establecer alternativas de acción.

En este contexto para definir alternativas de acción se requiere que la dirección desarrolle su capacidad emprendedora, al estimular los riesgos y las oportunidades y seleccionar entre diversas posibilidades de estrategias. Así mismo, que la dirección responda constantemente a las condiciones cambiantes del entorno y oriente a la organización hacia actividades empresariales adecuadas.

En relación a los planteamientos anteriores, Saloner et al. (2005) plantean que la planificación de escenarios es una herramienta estratégica por que le permite a los administradores determinar el desempeño probable de sus estrategias, realizar pruebas de su solidez y construir planes de contingencia.

En este sentido, refiere el autor que la meta en la planificación de escenarios es representar el futuro a través de unas cuantas descripciones. Según esto, las descripciones deben ser precisas y las variables que se usen para construirlas deben ser aquellas para las cuales la variación importante afecta el éxito de cualquier estrategia que persiga la organización. Si los escenarios son irrelevantes para el éxito estratégico de la empresa, serán inútiles en la evaluación de estrategias.

Bajo esta perspectiva, los administradores pueden evaluar el éxito de cada opción estratégica en cada escenario y, por ende, la fuerza de la estrategia para un rango de desarrollo posible en la industria. También pueden combinar esta información con la evaluación de la probabilidad de cada escenario para evaluar los riesgos y recompensas de una estrategia determinada. Desde este punto de vista, una estrategia bien orientada y que posea coherencia interna a menudo implicara hacer una gran apuesta en uno de los escenarios o en un pequeño subconjunto de estos.

En este orden de ideas, Madrigal (2002) expresa que puesto que el análisis de escenario impulsa al pensamiento creativo sobre el futuro, puede ser también una herramienta de interés en el desarrollo de la estrategia tecnológica al 
Planificación de escenarios: una herramienta estratégica para el análisis del entorno Yori Conill, Ligia; Hernández de Velazco, Judith y Chumaceiro Hernández, Ana

proveer tendencias y técnicas de simulación sustentados en la planificación estratégica.

Por otro lado, Illera (2005) señala que la estrategia evoluciona en forma constante en parte como respuesta a los cambios del entorno, otras veces como resultado de los esfuerzos de la dirección para crear nuevas oportunidades, ya que asumir los riesgos y aprovechar las oportunidades constituyen los retos de la actividad emprendedora de la dirección.

En virtud de los señalamientos expuestos, se puede afirmar que la planificación de escenarios constituye una herramienta estratégica, ya que esta se erige como un instrumento de planificación que permite medir y elegir entre opciones del desarrollo a través de la combinación de datos cualitativos y cuantitativos, permitiendo así optimizar el proceso de toma de decisiones al disminuir el nivel de incertidumbre presente en el medio ambiente externo.

En efecto, a través de esta técnica se intentan definir estrategias que puedan resultar eficaces cuando el futuro presenta un elevado nivel de incertidumbre. Así también, permite detectar y redefinir estrategias al cuestionar las tendencias y estrategias actuales de una manera eficaz, que las predicciones clásicas, mejorando la capacidad de pensamiento, dentro del ámbito de la planificación estratégica en cualquier organización.

\section{Utilidad de los escenarios dentro del contexto estratégico}

- La planificación de escenarios constituye una herramienta que permite mantener informado al gerente de los cambios suscitados a nivel de las variables macroeconómicas claves, entre ellas económicas, sociales, políticas, tecnológicas, gubernamentales, competitiva y demográficas, entre otras, que acontecen en el entorno las cuales provienen del análisis estratégico.

- Los escenarios constituyen una técnica de predicción cualitativa que permite predecir el comportamiento futuro de las variables ambientales claves $\mathrm{y}$, por ende, se erige como una herramienta de exploración del entorno a través del análisis minucioso del medio ambiente, el cual proviene del análisis estratégico.

- La planificación de escenarios constituye una de las técnicas mas populares dentro del ámbito de la predicción social que sirven de soporte a la planeación estratégica para optimizar la toma de decisiones y reducir el nivel de incertidumbre presente en el medio.

- Esta técnica permite identificar futuros posibles distintos dentro de un horizonte temporal con grandes probabilidades de ocurrencia, lo cual plantea consecuentemente la disyuntiva de elegir entre varias alternativas de acción erigiéndose esta en uno de los principales requisitos para generar estrategias.

- La planificación de escenarios permite construir representaciones de los futuros posibles, así como el camino que conduce a su consecución permitiendo establecer estrategias futuras.

- Esta técnica le permite a los gerentes determinar el desempeño de sus es- 
trategias a través de un proceso de monitoreo y evaluación de alternativas de acción, lo cual permite generar planes contingentes, incluso si la organización apuesta a ciertos escenarios, así también puede desarrollar planes acerca de qué hacer si aparece un escenario diferente y menos favorable.

- La planificación de escenarios posee una gran relevancia dentro del contexto estratégico, ya que provee un conjunto lógico de condiciones, tendencias y modelos de simulación provenientes de la planificación estratégica.

- Por otro lado, esta técnica impulsa al pensamiento creador y emprendedor, al permitir evaluar los riesgos y oportunidades contenidos dentro de las diversas alternativas o futuros posibles de ocurrencia, reconociendo que no todos los escenarios son igualmente probables y que el futuro puede en efecto tener aspectos de más de un escenario.

- Esta técnica también permite responder a las variaciones generadas en el medio ambiente, originando que las organizaciones asuman una actitud netamente proactiva y anticipatoria al orientar de manera significativa su destino hacia actividades empresariales adecuadas.

- La planificación de escenarios tiene un carácter flexible, al igual que la planificación estratégica, lo cual le permite adaptarse a los cambios generados en el entorno, a través de la utilización de planes alternativos o de contingencia.
- Esta técnica social no prevé lo que pasará, sino que describe futuros alternativos posibles con una determinada probabilidad de ocurrencia, que son de gran ayuda para el análisis estratégico.

- El proceso decisional dentro de la planificación de escenarios y las acciones que lo acompañan, buscan alcanzar una situación futura deseada, al igual que dentro del contexto de la planificación estratégica.

- La planificación de escenarios permite cuestionar las estrategias que han sido seleccionadas a través de la generación de un bosquejo de una situación concebible, dada una serie de supuestos.

- Esta técnica puede servir a varios fines, ya que proporciona un idioma común para el equipo de administración, y también brinda un medio para interpretar y comunicarse acerca de los acontecimientos nuevos, que afectan a la industria, ya que conforme se desarrollan los acontecimientos, ciertos escenarios se tornan más factibles que otros. Sin embargo, la virtud principal de los escenarios reside en la evaluación de opciones estratégicas potenciales.

- En este sentido, la planificación de escenarios puede incluir elementos anticipatorios que resultan difíciles de formalizar, tales como interpretaciones subjetivas de hechos, desplazamientos en valores, nuevas regulaciones o invenciones. También cabe destacar, que tanto para las situaciones de riesgo y de incertidumbre la planificación de escenarios es 
Planificación de escenarios: una herramienta estratégica para el análisis del entorno Yori Conill, Ligia; Hernández de Velazco, Judith y Chumaceiro Hernández, Ana

una herramienta de previsión de gran utilidad.

Con el propósito de justificar el objetivo planteado en la presente investigación y partiendo de la premisa de que todo individuo o sociedad tiene la capacidad latente de influir en la gestación de su propio futuro es que surge la necesidad de explorar el entorno a través de la aplicación de determinadas técnicas cualitativas exploratorias como es la planificación de escenarios.

Tomando como referencia los diversos enfoques generados y considerados en torno a la construcción de escenarios, los cuales presentan aspectos coincidentes y divergentes a seguir para la elaboración de escenarios, a continuación se proponen los siguientes pasos para la construcción de los mismos en función de la revisión realizada a los diversos enfoques de los autores anteriormente citados y bibliografías relacionadas.

Pasos para la construcción de escenarios:

1. Identificación del tema focal.

Se establece el título de lo que se pretende alcanzar, enmarcado dentro de un horizonte temporal determinado.

2. Definición del objetivo.

Este paso requiere el establecimiento del propósito básico del plan, planteando el objetivo en términos cuantificables o mensurables.

3. Descripción del presente.

Se realiza un planteamiento general de la problemática presentada, estableciendo las causas que originan la misma. También se describe el contexto ya sea político, económico, social, tecnológico y ambiental a través de la aplicación de diversas técnicas de recolección de datos ya sea mediante entrevistas, seminarios, Iluvia de ideas entre otras.

4. Definición del horizonte temporal. Se establece el plazo de ocurrencia del problema o evento objeto de estudio.

5. Identificación de los escenarios que se desean construir.

En este paso se muestran las múltiples opciones o conjeturas acerca de lo que puede suceder en función de la naturaleza de la problemática estudiada.

6. Identificación de las variables.

Se establece la relación entre las variables y las posibles consecuencias generadas producto de la interacción de las mismas las cuales pueden influir en el éxito o fracaso de la decisión a tomar.

7. Establecimiento de los actores.

En este paso se establecen los entes involucrados en la generación de la problemática planteada tanto en el presente como en el pasado, así como sus oportunidades estratégicas.

8. Determinar aquellos eventos que pueden ocurrir durante el espacio temporal de ocurrencia de los escenarios.

En este paso se prevén las discontinuidades que se puedan presentar en la construcción de los escenarios ya sean brechas o rupturas.

9. Construcción de los escenarios con su respectiva definición del horizonte temporal y la generación de un título de trabajo para cada escenario.

Se establecen representaciones de los futuros posibles así como el camino que conduce a su consecución. 
10. Definición de escenarios alternativos. Los cuales deben ser desarrollados en base a ciertos criterios tales como que los escenarios deben ser verosímiles, factibles, integradores, exhaustivos, comprensibles y deben estar ajustados a los objetivos previamente establecidos.

\section{Conclusiones}

Se puede concluir que la planificación de escenarios constituye una herramienta contenida dentro de las técnicas de predicción cualitativas, como un paso previo para desarrollar estrategias cuyo contenido debe ser elaborado con mucha precisión y coherencia con el fin de alcanzar resultados satisfactorios.

Las ideas expuestas confirman que los escenarios han de ser creíbles, comprensibles, consistentes, interesantes y exhaustivos. Además, deben ser concretos y concisos con el fin de no dispensar la información y servir de referente a la toma de decisiones, en la medida que le permita a la empresa prever las amenazas y oportunidades del entorno, con el fin de adaptarse a los cambios y situaciones contingentes y generalmente las organizaciones los utilizan para hacer flexible la planificación a largo plazo.

En el ámbito venezolano su utilización se limita a ciertos entes corporativos y algunos sectores gubernamentales, debido a la escasa visión de futuro y a la poca tradición en materia de planificación que poseen las empresas venezolanas, lo cual inhibe la variabilidad de las propuestas de estrategias desde diferentes situaciones hipotéticas del entorno. No obstante, actualmente algunas universi- dades y centros de investigación están propugnando su utilización a través de sus programas de educación, valiéndose del desarrollo de las tecnologías de información.

Cabe señalar, que esta técnica es un recurso de cálculo costoso ya que resulta necesario desarrollar un plan de contingencia para enfrentar cada escenario, por ello es frecuente que se utilicen muy escasas y a veces una sola variable y que se fije para ella un valor pesimista o de piso y un valor optimista o de techo. En el caso que la empresa no cuente con las competencias necesarias para manejar este proceso resulta recomendable recurir a consultores especializados.

En este sentido, se sugiere ampliar la utilización de esta herramienta de predicción social, con el fin de ampliar los conocimientos del entorno y anticipar las amenazas y oportunidades del mismo, lo cual permitirá a las empresas ampliar su visión de futuro a través del desarrollo de alternativas o cursos de acción orientados a reducir la incertidumbre y optimizar la toma de decisiones.

En síntesis la técnica de escenarios se presenta como una herramienta capaz de orientar la gestión organizacional a través de la detección de futuros probables y la determinación de alternativas de actuación. Es pues, más que una herramienta de predicción, una herramienta de anticipación destinada a la reducción de incertidumbre y la minimización del costo de oportunidad que conlleva al diseño de un plan estratégico.

En efecto, a través de esta técnica se definen estrategias que pueden resultar eficaces cuando el futuro presenta un fuerte nivel de incertidumbre. De igual manera, se 
Planificación de escenarios: una herramienta estratégica para el análisis del entorno Yori Conill, Ligia; Hernández de Velazco, Judith y Chumaceiro Hernández, Ana

recomienda su correcta aplicación para la resolución de los asuntos públicos, tanto en el contexto nacional, como regional y local, considerando las predicciones sociales para una oportuna, acertada, incluyente y participativa toma de decisiones en la construcción del bien común.

\section{Referencias bibliográficas}

Bas, Enric (2002). Prospectiva. Como usar el pensamiento sobre el futuro. Barcelona. España. Editorial Ariel, S.A.

Certo, Samuel y Peter, Paul (1996). Dirección Estratégica. Primera edición española de la tercera edición en inglés. Madrid. España. Producción editorial.

David, Fred (2003). Conceptos de Administración Estratégica. Novena Edición. Impreso en México. Editado por Pearson educación de México, S.A. de CV.

Dess, Gregory y Lumkin, Gregory (2003). Dirección Estratégica. España S.A. Madrid. Primera Edición. McGraw Hill Interamericana.

Duane, David (2001). Investigación en Administración para la Toma de Decisiones. S.A. Argentina. Quinta Edición Internacional. Thompson Editores.

Francés, Antonio (2001). Estrategia para la empresa en América Latina. Caracas Venezuela. Ediciones lesa.
Garrido, Santiago (2003). Dirección Estratégica. McGraw Hill Interamericana de España S.A. Madrid.

Garrido, Santiago (2006). Dirección Estratégica. Edición. Madrid España. Editorial McGraw Hill, segunda.

Hitt Michael, Ireland Duane y Hoskisson Robert (2004). Administración Estratégica. S.A. Impreso en México. Internacional Thompson Editores.

Illera, Luis (2005). Política Empresarial. Línea de Dirección y Estrategias. Primera Edición. Impreso en Colombia. Coedición CESA y Mayol Ediciones, S.A.

Madrigal, Bertha (2002), Habilidades Directivas. McGraw Hill - Impreso en México. Interamericana. Editores. S.A.

Saloner Garth, Shepard Andrea y Podolny Joel (2005). Administración Estratégica. Editores México. DF. Editorial Limusa, S.A. de CV Grupo de Noruega.

Serna, Humberto (2001). Planeación y Gestión Estratégica. Cuarta edición. Bogota. Colombia. Ram Editores. C.A. LTDA.

Thompson, Arthur; Strickland Albert (2004). Administración Estratégica. Impreso en México. McGraw Hill - Interamericana Editores, S.A. DE. C.V.

www.cendes-ucv.edu.ve/docencia-detaye. jsp.id=69 (14-02-2009)

www.fudeco.gob.ve/proyectos/pdf/indice3.pdf (08-04-2009) 